\title{
Diclofenac potassium/nitazoxanide/azithromycin novel COVID-19 protocol used in adults, children and pregnant patients: could it be the magic bullet?
}

\author{
Mina Kelleni ${ }^{1}$ \\ ${ }^{1}$ Affiliation not available
}

December 14, 2020

\begin{abstract}
Background: The current pandemic of coronavirus disease 2019 has necessitated trial of several drugs searching for a potential cure. We have published an article to justify adoption of nitazoxanide/azithromycin for early cases of COVID-19 followed by two other articles providing the theoretical basis to add non-steroidal anti-inflammatory drugs to the protocol which is described in this manuscript.

Patients and methods: Twenty-two consented confirmed and highly suspected COVID-19 Egyptian patients including 11 adult males, 6 adult females, 2 pregnant patients as well as 3 children. All patients have received a 5-day-regimen of NSAIDs (diclofenac potassium, ibuprofen or ketoprofen)/nitazoxanide/azithromycin either in full or in part as illustrated in the manuscript. The primary endpoint of this protocol was full relief of all significant/hazardous COVID-19 symptoms and signs like fever, progressive cough, moderate/severe dyspnea or disturbed level of consciousness.

Results: The primary endpoint of this protocol was fully achieved in all patients within two weeks. Most of the patients treated early with the protocol have recovered during the five days; the lymphocytic count was significantly improved for those with prior lymphopenia. No significant adverse effects were reported.

Conclusion: A novel short course COVID-19 protocol using inexpensive FDA approved drugs is illustrated.
\end{abstract}

COVID-19 original research article

Diclofenac potassium/nitazoxanide/azithromycin novel COVID-19 protocol used in adults, children and pregnant patients: could it be the magic bullet?

Mina T. Kelleni, MD, PhD

Assistant Professor of Pharmacology, College of Medicine, Minia University, Egypt.

Mobile: +201200382422

drthabetpharm@yahoo.com, mina.kelleni@mu.edu.eg

https://orcid.org/0000-0001-6290-6025

Keywords: COVID-19, Diclofenac potassium, Ibuprofen, Nitazoxanide, Azithromycin.

Abstract diagram 


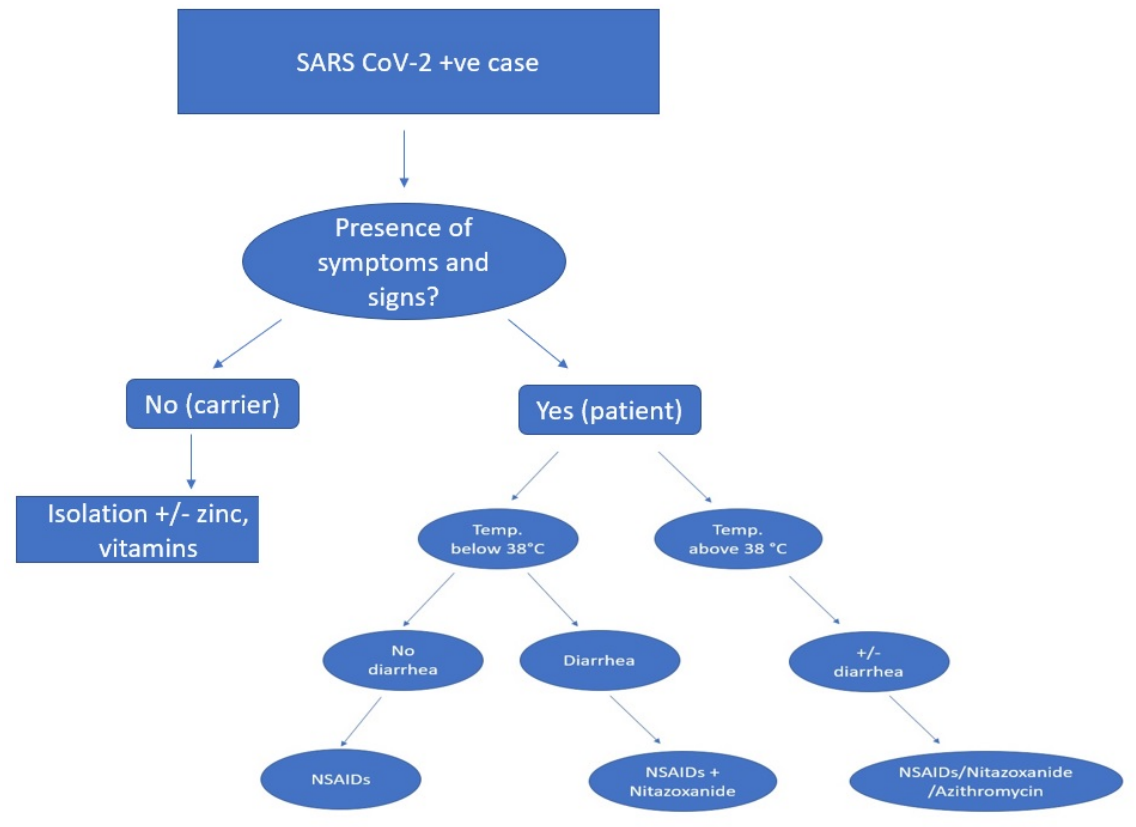

A step by step novel protocol to manage SARS CoV-2 positive cases.

\section{Introduction}

Corona virus disease 2019 (COVID-19) is considered one of the worst pandemics in modern history with regard to its rapid rate of spread which has infected more than 69 million persons and globally harvested more than a million and five hundred thousand lives as of the $10^{\text {th }}$ of December 2020 [ COVID-19 Dashboard by the Center for Systems Science and Engineering (CSSE) at Johns Hopkins University]. Keeping in mind that a vaccine might not be the ultimate safe, rapid and effective solution to compete with the huge global morbidity and mortality caused by the highly evolving severe acute respiratory syndrome coronavirus 2 (SARS CoV-2) ${ }^{1-4}$, the author, a physician as well as a pharmacologist, has searched for effective drugs that might provide a cure and published an early manuscript providing the scientific pathophysiological and pharmacological concept to use the inexpensive, safe drug novel combination; nitazoxanide/azithromycin for early management of COVID-19 at a time when only eight clinical trials, with recruitment difficulties, for nitazoxanide potential in COVID-19 were undergoing in developing countries ${ }^{5}$. Noteworthy, as of the $10^{\text {th }}$ of December 2020, the number of these trials has reached 24 registered clinical trials in various countries including five recruiting clinical trials in the USA and two completed studies in Brazil ${ }^{6}$. Moreover, an openlabel observational study has recently recommended that some drug combinations including azithromycin and nitazoxanide should be considered for those diagnosed early with COVID-19. Moreover, they have opted for nitazoxanide among those combinations, due to more extensive demonstration of in vitro and in vivo antiviral activity, proven efficacy against other viruses in humans, and steadier safety profile ${ }^{7}$.

We have subsequently managed to publish a preprint ${ }^{8}$ to suggest adding non-steroidal anti-inflammatory drugs (NSAIDs) such as ibuprofen for early management of COVID-19. Importantly in this preprint, that has been later published ${ }^{9}$, we have suggested a novel lymphocyte distraction hypothesis to explain the pathogenesis of COVID-19 in general and its subsequently reported COVID-19 multisystem hyper-inflammatory syndrome in children. Based on the suggested hypothesis, we suggested that NSAIDs will be of COVID-19 value not only as safe and more effective analgesic antipyretic but also, due to its potent anti-inflammatory effect, might reverse the pathogenesis of COVID-19, prevent its complications and improve the clinical outcomes especially if used as early as possible during the course of COVID-19. This later suggestion has contradicted the more prevalent approach adopted worldwide that has avoided NSAIDs and, unfortunately, 
preferred paracetamol that does not possess major peripheral anti-inflammatory effects as compared to NSAIDs ${ }^{10}$. Notably, we have fully explained from a pharmacological point of view the very unfortunate incident that led to the avoidance of the use of ibuprofen in COVID-19 ${ }^{11}$, other researchers have also criticized avoiding ibuprofen in COVID-19 12 and ibuprofen use was not shown to be associated with worse COVID-19 clinical outcomes, compared with paracetamol or no antipyretic ${ }^{13,14}$. Moreover, ibuprofen has been shown to be among the drugs that are significantly associated with diminished COVID-19 risk for hospitalization as revealed by an In silico cohort study that examined the electronic health records from individuals 7,360 individuals with COVID-19 positive test results by PCR. Further, ibuprofen and naproxen, which are both among NSAIDs, were more commonly prescribed among individuals not requiring intensive care ${ }^{15}$. Notably, two registered recruiting clinical trials to test the role of ibuprofen in COVID-19 are undertaken; one in the UK testing lipid ibuprofen (NCT04334629) and the other to test experimental inhaled hypertonic ibuprofen in Argentina (NCT04382768).

\section{Study design}

Egypt is considered one of the highest African countries with regards to the number of COVID-19 fatalities compared to that of infected cases; 119,702 infections and 6,832 coronavirus-related deaths as of December 10, $2020^{16}$. Since late April 2020, we have strongly, promptly and publicly through YouTube videos, Facebook posts and local newspapers criticized the use of hydroxychloroquine, oseltamivir and lopinavir-ritonavir and remdesivir in COVID-19 protocols ${ }^{17}$, considering them as possible causes for the declared high mortality rate encountered in Egypt, a criticism attributed to their lack of efficacy that was being revealed later ${ }^{18-21}$. The author, while working in KSA, has received compassionate requests and consents for treating Egyptian patients since the $13^{\text {th }}$ of May 2020 and the last patient reported in this manuscript has been enrolled on the $19^{\text {th }}$ of October 2020 after his return to Egypt. We have responded immediately to the received requests from patients and a personalized fully documented telemedicine approach, using the author's Facebook messenger account and WhatsApp, was used and we have used the described protocol using NSAIDs, nitazoxanide, azithromycin +/- cefoperazone in full or part as described later for those patients knowing that all the used drugs are relatively safe and potentially more effective as compared to the used drugs used in $\mathrm{MOH}$ criticized protocols. Notably, antibiotics are liberally purchased without a formal prescription in Egypt. The primary endpoint of this study was full relief of COVID-19 induced hazardous, i.e. other than anosmia and/or ageusia, symptoms and signs e.g. fever, progressive cough, moderate/severe dyspnea and/or disturbed level of consciousness.

\section{Patients and Eligibility}

The patients included a nurse who has received a verbal, the routine Egyptian procedure, $\mathrm{MOH}$ confirmation of a positive SARS CoV-2 PCR; a pregnant patient who has been initially confirmed with SARS CoV-2 IgM rapid test and later confirmed by MOH PCR; a severe COVID-19 female patient confirmed by both CT and rapid IgM test; a moderate COVID-19 male patient confirmed by CT; 2 male Egyptian patients who tested positive for SARS CoV-2 by PCR who adopted our advice for treatment while in quarantine in KSA; a male Egyptian patient living in KSA who tested positive by PCR and received the treatment while isolated at home after a change in quarantine policy in KSA, a few days later, his wife developed COVID-19 potential manifestations e.g., fever, cough, malaise, anosmia and ageusia only after an early PCR test revealed negative results and she couldn't repeat it and she received the treatment like her husband and an Egyptian patient working in KSA who was also diagnosed with COVID-19 and confirmed by PCR. The other 13 highly suspected cases, including another pregnant patient as well as three children, have presented a combination of some or all of the following: history of close contact to first degree relatives/ close associates COVID-19 quarantined/isolated patients; clinical manifestations suggestive of COVID-19 e.g. fever, cough, dyspnea, anosmia and/or ageusia; CBC showing leucopenia or lymphocytopenia with no ability to perform rapid Ig $\mathrm{M}$ test or PCR test which are not readily available in Egypt. Importantly, the clinical picture as well as the investigations differed from one patient to another and we used an individualized medical approach for each case, e.g., most patients who have performed CBC showed lymphopenias while some have showed marginal "apparently" normal complete blood count but showed history and clinical manifestations highly suggestive 
of COVID-19. No restrictions for selection of patients were present. However, we refused to manage some cases because those who contacted the author had no direct contact with the patients and thus no close follow up was guaranteed. The author has insisted to contact only the case/legal guardian and only in one case the husband and sons of a deteriorating patient who couldn't give an initial consent as described later.

\section{NSAIDs might play an integral role in managing early COVID-19}

Interestingly, the nurse with the verbal Egyptian MOH confirmation of positive SARS CoV-2 PCR had a total white blood cell count of $7.8 \times 10^{3} / \mu$ l, relative lymphocyte count $5.5 \%$, mixed population of monocytes, basophils and eosinophils count $16.5 \%$ and neutrophils count $78 \%$ (Figure 1). The swab revealed positive on the $18^{\text {th }}$ of May, 2020 and he started to take the ibuprofen/nitazoxanide/azithromycin protocol on the $19^{\text {th }}$ of May and after the 5 day treatment regimen, the total white blood cell count was $6.3 \mathrm{X} 10^{3} / \mu$, lymphocyte count $43 \%$, mixed population of monocytes, basophiles and eosinophils count $8 \%$ and neutrophils $49 \%$ (Figure 2) and this significant elevation of lymphocytes might be considered as a preliminary clinical proof of the suggested COVID-19 pathogenesis theory ${ }^{9}$. The significant decrease of neutrophils may represent the efficacy of azithromycin in eliminating any associated secondary bacterial infection. Notably, we suggested adopting larger randomized clinical trials, as described in two published manuscripts, for the drugs mentioned in this protocol as well as other suggested drugs ${ }^{5,8}$.

Moreover, an Egyptian expat in KSA with SARS CoV-2 PCR positive test results of a combined nasopharyngeal and oropharyngeal swab was also a hypertensive and type 2 diabetic patient and managed, upon advice, to receive diclofenac potassium to replace the prescribed paracetamol. This patient has already received amoxicillin/clavulanate potassium and thus, we decided not to administer any further antibiotics as no fever was detected. Interestingly, his troublesome fortnight dyspnea and dry cough were reported to dramatically improve from the first day diclofenac potassium $50 \mathrm{mg}$, postprandial b.i.d., was administered and he became mostly symptom-free in three days. The patient continued further two days and later he was discharged. Notably, he was advised, similar to our other COVID-19 patients, not to take antitussives for his mild to moderate cough episodes, and to use warm beverages e.g. boiled mint to soothe their sore throat and this approach has proved beneficial in all our cases, it's been a recommendation basing on a clinical sense that trusted the body natural reflexes and was not basing on a scientific evidence based evidence, which is currently lacking and might not be promptly available. For the second isolated expat, we prescribed diclofenac potassium/azithromycin to manage his persistent 20 days fluctuating fever. Notably, this patient managed to get azithromycin with a help from an Egyptian pharmacist as antibiotics are not allowed to be purchased without a prescription in KSA and he reported that his persistent diarrhea started to improve immediately after ceasing to use the previously prescribed antitussive dextromethorphan even before managing to purchase the other newly prescribed drugs from outside the quarantine facility. Furthermore, he has also used ibuprofen $600 \mathrm{mg}$ tablets that were available with him and reported a better clinical experience than diclofenac potassium regarding headache, fatigue and pain control and later he was discharged. Importantly, we preferred to give NSAIDs twice daily aiming at improving the natural immune response if the patients recorded temperature less than $38^{\circ} \mathrm{C}$ in between the two doses but they were allowed to take them t.d.s if the temperature exceeded $38^{\circ} \mathrm{C}$ before the next dose. Similarly, we prescribed diclofenac potassium/azithromycin to safely manage a third expat patient, also confirmed by PCR, who complained of $39^{\circ} \mathrm{C}$ fever associated with his SARS CoV-2 infection and a fourth COVID-19 expat patient also confirmed by PCR, suffered from fluctuating fever up to $39^{\circ} \mathrm{C}$ with marked dry cough to which he was prescribed azithromycin $500 \mathrm{mg}$ daily for three days and paracetamol. We, contacted on the third day, instructed him to continue azithromycin for 2 more days and to replace paracetamol with diclofenac potassium t.d.s. for two days and b.i.d. for three days and he felt marked improvement in his clinical condition with normal temperature and gradual significant improvement of cough during the five days course.

Notably, nitazoxanide is not commercially available in KSA pharmacies but is readily available in Egyptian pharmacies. Some patients have also complained of severe malaise, anorexia, moderate diarrhea, anosmia, dysgeusia, ageusia, moderate to severe flank and back pain which have been totally improved in two weeks. Most of the patients who received the protocol early in COVID-19 have improved during the its five-day 
duration. Notably, some young individuals were close contacts to positive COVID-19 cases with no or very mild symptoms e.g. mild cough without fever and for those suspected silent carriers, only isolation $+/$ - zinc and vitamins was advised and reassurance was granted with their smooth follow up.

\section{The protocol has been shown to be effective and safe for three children and two pregnant patients}

Furthermore, the patients included 2 pregnant patients; one in her $9^{\text {th }}$ week of gestation (confirmed by SARS CoV-2 rapid IgM test and later by Egyptian $\mathrm{MOH}$ PCR) and the other is a highly suspected case in her $18^{\text {th }}$ week. They also have included 2 female children, 6 years and eight months- and 1.5-year-old sisters and a 4-year-old boy.

The pregnant patient who was confirmed by PCR has been also treated for rheumatoid arthritis and her COVID-19 clinical picture included fever, sore throat, fatigue and dry cough. We stopped both hydroxychloroquine and sulfasalazine already used for rheumatoid arthritis during the five-day treatment course to prevent potential adverse drug interactions and we successfully used azithromycin/nitazoxanide for her treatment. Similarly, NSAIDs were not prescribed for her as she was already on prednisone for her rheumatoid arthritis. Several prenatal four-dimensional ultrasonography that followed the treatment course showed a healthy female fetus with no suspected congenital abnormalities and on the $10^{\text {th }}$ of December 2020, she delivered a full term healthy female offspring. The other pregnant was in her $18^{\text {th }}$ week of gestation and was highly suspected, by history of close contact to a positive COVID-19 case, clinical manifestations of sore throat, cough and declining lymphocytic titer (24\%) as compared to a previous CBC (39\%) and she completed one-week diclofenac potassium $50 \mathrm{mg}$ b.i.d. course though she was strongly recommended to use it for only five days. However, she's been totally relieved of COVID-19 symptoms with no significant adverse effects reported and she later delivered a full term healthy female offspring. We suggest that the potential benefits are exceeding the risks for this short-term NSAIDs regimen for pregnant patients before the third trimester, and we followed each case eagerly providing a personalized prescription. Similarly, we assessed any possible contraindication or potential drug-drug interactions.

Importantly, we prescribed the pediatric weight/age adjusted doses of nitazoxanide suspension, azithromycin suspension and ibuprofen syrup as illustrated. The mentioned three children were highly suspected by history of a close contact to a positive COVID-19 patient, clinical picture including fever, sore throat and/or cough. They recovered smoothly within the five-day regimen with no reports of adverse effects. Moreover, the elderly child complained of severe sore throat that led her to severe anorexia, while complaining of diarrhea and vomiting with a risk for dehydration while on paracetamol and she as well as her sister, complaining of milder manifestations, had completely recovered from their symptoms in three days but continued the five day full regimen and we suggest that the relief of the severe sore throat that restored the appetite might be due to the action of both ibuprofen and azithromycin. Notably, in other less documented suspected COVID-19 children, not mentioned in this manuscript, complaining of moderate cough episodes, we also prescribed the locally available antitussive suppositories/syrup containing oxomemazine, guaiphenesin, sodium benzoate and paracetamol once at night to allow a better sleep with no reported adverse effects.

\section{A modified version of this protocol managed two complicated COVID-19 patients}

Interestingly, a 48-year-old female patient complained of severe sore throat, malaise, fever and mild cough to which a family physician who is a tropical medicine consultant has asked for a CT chest that revealed patchy ground glass opacities scattered at right lung mainly peripheral in location associated with minimal thickening of the lung interstitium as well as peribronchial cuffing suggestive of COVID-19 (Figure 3), as described in the given report, and her family physician has prescribed paracetamol, betamethasone injection IM, doxycycline $100 \mathrm{mg}$ for 15 days, azithromycin $500 \mathrm{mg}$ orally, some vitamins and minerals as well as a syrup that contains antihistaminic and expectorant for three days. Her condition rapidly deteriorated with persistent fever, yellow vision, tachycardia and marked confusion. When we received a compassionate request from her husband and sons to help, we asked for an urgent CBC (Figure 4) that revealed a total leucocytic count of $5.1 \times 10^{3} / \mu$ with a low lymphocytic count of $12 \%$. She was diagnosed positive for SARS CoV-2 
IgM upon our request and we immediately stopped the bacteriostatic doxycycline, and ordered an immediate ketoprofen IM injection to be followed by diclofenac potassium b.i.d. and azithromycin $500 \mathrm{mg}$ once daily for five days in combination with empiric parenteral cefoperazone; chosen for its readily availability in Egypt and its broad spectrum antibacterial efficacy against atypical respiratory micro-organisms that might be sharing in causing the high fever, $1 \mathrm{gm}$ once daily for the first three days. Notably, the author has chosen a pharmacovigilant low dosed regimen of cefoperazone to avoid the very rare potential of vitamin K-dependent coagulopathy which might develop with serious diseases ${ }^{22}$, considering that the author was not in direct contact with patient as well as that cefoperazone was given in association with full 5-day-course of azithromycin. Interestingly close monitoring for potential increase of cefoperazone dose but it has not been needed. We have also added nitazoxanide $500 \mathrm{mg}$ b.i.d. that also managed to control an episode of diarrhea that has been encountered after the start of treatment. We also stopped the previously prescribed antitussive syrup as explained earlier. Her condition improved gradually yet dramatically within five days and the $\mathrm{CBC}$ on the fifth day (figure 5) revealed an improved total leucocytic count of $6 \mathrm{X} 10^{3} / \mu$ l with a significantly elevated lymphocytic count of $23 \%$. She returned almost normal within two weeks complaining only of a mild cough and insignificant chest tightness that have disappeared later spontaneously.

Similarly, a 45-year-old male with clinical picture suggestive of mild-moderate COVID-19 including fever up to $39^{\circ} \mathrm{C}$ has received a protocol of azithromycin, paracetamol, zinc and vitamin $\mathrm{C}$ for only three days to which his body temperature fluctuated between $37^{\circ} \mathrm{C}$ and $38^{\circ} \mathrm{C}$. However, a more severe clinical picture of high fever, sever fatigue, profuse sweating, severe episodes of cough with newly developed dyspnea has developed after two days of stopping treatment and we have received a request to treat on that fifth day. CT has confirmed a viral pneumonia with a bilateral scattered subpleural and parenchymal patches of ground glass opacities suggestive of COVID-19 for clinical and laboratory correlation (Figure 6) and we prescribed parenteral cefoperazone once daily for three days with another course of azithromycin $500 \mathrm{mg}$ once daily for five days as well as oral diclofenac potassium t.d.s for the first two days followed by b.i.d. for three other days when his condition started to improve and we have not prescribed nitazoxanide for this patient waiting for the initial clinical response. The patient has experienced a significant clinical improvement as regards to most of his symptoms with return of body temperature to normal with no additional antipyretic or antibiotics after the new 5-day-course. He has also experienced gradual improvement of his severe cough and got free of its severe episodes after seven days with no antitussive prescribed as discussed before and returned almost normal as regards to cough, after 2 weeks. Interestingly, a follow up CT performed in another center one week after the first one has revealed regressive course of viral pneumonia with residual bilateral scattered patches of ground glass opacities according to its report (Figure 7). Moreover, though the lymphocytic count of this patient before treatment was $30 \%$ and not considered lymphopenic (Figure 8), it has increased to $47.9 \%$ (Figure 9) after the 5-day-course of treatment raising another possibility that it might have been deteriorating and the NSAIDs therapeutic intervention has efficiently stopped and reversed this deterioration as hypothesized before ${ }^{9}$. Notably, this patient $\mathrm{C}$ reactive protein level was $54 \mathrm{mg} / \mathrm{l}$ at the start of treatment and has been elevated to $90 \mathrm{mg} / \mathrm{l}$ at the end of the 5 -day-course and one week after its end, the level has decreased to $9 \mathrm{mg} / \mathrm{l}$. The initial increase in CRP might be explained by an undergoing concomitant bacterial infection that has been treated efficiently by the administered antibiotics. Further, this patient was suffering from type 1 diabetes mellitus for 8 years and he has experienced a significant deterioration of his blood glucose profile to which we increased the total daily biphasic isophane insulin dosage with new addition of insulin glargine for two weeks.

\section{Pharmacoeconomics and pharmacovigilance are respected in telemedicine}

We were waiting for the negative SARS CoV-2 (COVID-19) qualitative PCR test confirmation from the Egyptian expats living in KSA to develop a personal view for the best period of isolation but because of the large numbers of new infections, it was decided not to repeat the test after the clinical improvement and we agree with this pharmacoeconomically vigilant attitude. However, we recommended all the encountered Egyptian patients to remain isolated for 21 days from the beginning of the symptoms including at least one week, better ten days, of a totally symptom free period even if the PCR test revealed negative earlier and this recommendation was to avoid the possibility of any false negative results in Egypt. This advice was also 
based on a positive test from the nurse, although he was symptom free for more than 10 days to be fully elucidated by other research work to determine the best possible recommendation for the isolation period. We know that this advice for the isolation period of Egyptian patients is not consistent with the mainstream current guidelines, yet we chose to recommend more rest, if possible, especially as isolation rules are still changing ${ }^{23}$ to be noted that a study has recommended a 22 -day quarantine period to avoid the $6.7 \%$ failure who showed symptoms after the 14-day quarantine period; the 22-day quarantine showed a failure rate below $1 \%$ with $95 \%$ confidence ${ }^{24}$.

\section{A roadmap to combat COVID-19 using inexpensive available FDA-approved drugs}

For adults, we used NSAIDs, either postprandial ibuprofen $400 \mathrm{mg}$ (600 mg in KSA) b.i.d. or diclofenac potassium $50 \mathrm{mg}$ b.i.d. alone for early cases complaining of sore throat, dry cough or mild dyspnea with no fever or fever less than $38^{\circ} \mathrm{C}$ and we believe they have helped to prevent the progression of COVID-19 especially for those patients who received them earlier. However, we recommend adding nitazoxanide, when available, to NSAIDs for patients complaining from diarrhea even if their temperature wasn't above $38^{\circ} \mathrm{C}$ and it has also proved effective, as part of the whole protocol, to control a moderate diarrhea encountered in an adult patient whose temperature exceeded $38^{\circ} \mathrm{C}$. Moreover, we prescribed the whole NSAIDs (as described)/nitazoxanide (500 mg bid)/azithromycin (500 mg once daily) five days protocol for COVID-19 adult patients complaining of fever more than $38^{\circ} \mathrm{C}$. Importantly, other than their suggested potential curative COVID-19, we also noticed that NSAIDs had remarkably superior symptomatic clinical efficacy compared to paracetamol, for those who used paracetamol before switching to the new protocol, for controlling high fever, headache and malaise.

Notably, we allowed all patients to continue the already prescribed vitamin C, Vitamin D, zinc, lactoferrin or any other food supplements, although their proven clinical benefit, if any, are yet to be proved. We suggest these supplements when administered in their proper dosage are almost harmless regardless if they might or might not prove beneficial synergistic effect.

\section{Confirmations and limitations}

We would also like to declare that we believe that the drugs mentioned in this protocol are being used by other physicians in multiple parts of the world away from the clinical trials and sometimes without the scientific basis that explains their efficacy, this basis, we believe we have provided in our published manuscripts. We have been informed by an Egyptian colleague that he has used this preprinted protocol ${ }^{25}$ effectively and safely to treat more than 15 COVID-19 patients and that he has also used another NSAIDs; celecoxib to manage pyrexia encountered in some of his patients with similar positive results. Notably, celecoxib, when even used as adjuvant therapy, has been shown to be beneficial for COVID-19 patients ${ }^{26}$. Similarly, a Mexican pulmonologist has sent a letter, after publishing the nitazoxanide/azithromycin protocol paper, confirming his successful practice managing 24 COVID-19 patients with nitazoxanide and complaining from a lack of protocol. A link to the full preprinted protocol has been sent to him. However, we had no funding to perform double blind randomization against the standard care protocol or to perform extensive and well organized investigations for each patient, many with limited economic resources, and these important limitations need to be addressed by other researchers with better capabilities to build on this protocol and release their results and/or modifications.

\section{Conclusion}

Finally, we wish to declare that the described short novel protocol, including NSAIDs as integral component, has shown a prompt efficacy, full compliance, tolerability and no reported serious adverse effects. Further, we wish to suggest that it is an effective one using inexpensive, safe and FDA approved drugs might be readily available, wishing that the time to adopt our suggested positive recommendation to use of NSAIDs as early as possible in the course of COVID-19 might be adopted soonest.

\section{Ethics and consent to participate}


The author is a practicing member of the Egyptian syndicate of physicians since 2006. Every ethical command has been followed in all procedures mentioned in this manuscript.

Consents, verbal and later confirmed by written conversations through the mentioned social media applications, were obtained from each case/legal guardian involved in this study who have also authorized the release of their laboratory/radiological results.

The author was working during most of this study in KSA and the preprint was published while he was working there. I have returned to Egypt after gratefully resigning from my Saudi University to return to my home country and patients on the $22^{\text {nd }}$ of September 2020. Basing on an early knowledge represented later in published peer-reviewed articles; a moral obligation and oath to serve and help led to an immediate reply and thus getting consents from the mentioned Egyptian patients; most of them were residing in Egypt and the expat ones were either isolated or dwelled away from my province, was the only available method to conduct the current study.

\section{Availability of data and materials}

The author declares full transparency and all data and materials related to this study are available. All the findings, other than investigation reports, were documented mostly in Arabic; the mother tongue of the patients as well as the author.

\section{Conflict of interest}

The author declares there're no conflicts of interest.

\section{Funding/Financial exposure}

None.

\section{Acknowledgment}

All the mentioned medical consultations were performed free of charge (Mat 10:8) and I pray that the Lord Almighty who guided my humble steps through the unimaginable hardships of publishing each of my COVID-19 articles, might use them for the glory of His holy name through saving precious lives; this my foremost wish. The author would like to sincerely thank all the Egyptian patients who participated in this study as well as their family members who watched their treatment. Also, many thanks to Dr. Haroun Akhnoukh and Dr. Pablo Juárez for their precious feedback.

\section{References:}

1. The First Covid Vaccines May Not Prevent Covid Infection (1). 2020, June 15 . at https://news.bloomberglaw.com/coronavirus/the-first-covid-vaccines-may-not-prevent-you-fromgetting-covid?context $=$ search\&index $=15 \&$ utm_source $=$ twitter\&campaign $=$ B1BDE4E0-AF5C-11EAA029-9CF94F017A06\&utm_medium=lawdesk\&fbclid=IwAR3HM9yKf4JxM99n0ZRqCd-QqFSnWOcTc8T1NvOGnN1f-WaD3Ip0DCmBks.)

2. Ibarrondo FJ, Fulcher JA, Goodman-Meza D, et al. Rapid Decay of Anti-SARS-CoV-2 Antibodies in Persons with Mild Covid-19. New England Journal of Medicine 2020.

3. Fauci warns COVID-19 vaccine may be only partially effective, public health measures still needed. 2020, August 7. at https://www.reuters.com/article/us-health-coronavirus-fauci-vaccine/fauci-warns-covid19-vaccine-may-be-only-partially-effective-public-health-measures-still-needed-idUSKCN2532YX.)

4. SARS CoV-2 adenovirus and RNA based vaccines potential autoimmune complications: could we lower the chances? Authorea, 2020, November 30. at https://www.authorea.com/users/318758/articles/496102-sarscov-2-adenovirus-and-rna-based-vaccines-potential-autoimmune-complications-could-we-lower-the-chances.)

5. Kelleni MT. Nitazoxanide/Azithromycin combination for COVID-19: A suggested new protocol for COVID-19 early management. Pharmacological Research 2020:104874. 
6. 24 Studies found for: Nitazoxanide | Covid19. 2020, December 10 . at https: $/$ clinicaltrials.gov/ct2/results?cond $=$ Covid19\&term $=$ Nitazoxanide\&cntry $=\&$ state $=\&$ city $=\&$ dist $=$.)

7. Cadegiani FA, Goren A, Wambier CG, McCoy J. Early COVID-19 Therapy with Azithromycin Plus Nitazoxanide, Ivermectin or Hydroxychloroquine in Outpatient Settings Significantly Reduced Symptoms Compared to Known Outcomes in Untreated Patients. medRxiv 2020:2020.10.31.20223883.

8. Ibuprofen potential addition to COVID-19 early management protocols: could it be superior to paracetamol and hydroxychloroquine? 2020, May 6 . at https://journal.sketchingscience.org/users/318758/articles/448656-ibuprofen-potential-addition-to-covid19-early-management-protocols-could-it-be-superior-to-paracetamol-and-hydroxychloroquine.)

9. Kelleni MT. Early use of non-steroidal anti-inflammatory drugs in COVID-19 might reverse pathogenesis, prevent complications and improve clinical outcomes. Biomedicine \& Pharmacotherapy 2021;133:110982.

10. Anderson BJ. Paracetamol (Acetaminophen): mechanisms of action. Pediatric Anesthesia 2008;18:915-21.

11. Kelleni MT. ACEIs, ARBs, ibuprofen originally linked to COVID-19: the other side of the mirror. Inflammopharmacology 2020.

12. Moore N, Carleton B, Blin P, Bosco-Levy P, Droz C. Does Ibuprofen Worsen COVID-19? Drug safety 2020;43:611-4.

13. Rinott E, Kozer E, Shapira Y, Bar-Haim A, Youngster I. Ibuprofen use and clinical outcomes in COVID19 patients. Clinical Microbiology and Infection.

14. Lund LC, Kristensen KB, Reilev M, et al. Adverse outcomes and mortality in users of non-steroidal anti-inflammatory drugs who tested positive for SARS-CoV-2: A Danish nationwide cohort study. PLoS Med 2020;17:e1003308.

15. Castro VM, Ross RA, McBride SMJ, Perlis RH. Identifying common pharmacotherapies associated with reduced COVID-19 morbidity using electronic health records. medRxiv 2020:2020.04.11.20061994.

16. Egypt COVID-19 TRACKER. 2020, December 10. at https://graphics.reuters.com/world-coronavirustracker-and-maps/countries-and-territories/egypt/.)

17. Remdesivir is least likely to be effective for safe treatment of COVID-19: A pharmacovigilant point of view Authorea, 2020, December 01. at www.authorea.com/496404/7jNIUYA0Nj7hHkx9PC6_lQ.)

18. Tan Q, Duan L, Ma Y, et al. Is oseltamivir suitable for fighting against COVID-19: In silico assessment, in vitro and retrospective study. Bioorg Chem 2020;104:104257-.

19. Pan H, Peto R, Karim QA, et al. Repurposed antiviral drugs for COVID-19 -interim WHO SOLIDARITY trial results. medRxiv 2020:2020.10.15.20209817.

20. Horby PW, Mafham M, Bell JL, et al. Lopinavir-ritonavir in patients admitted to hospital with COVID19 (RECOVERY): a randomised, controlled, open-label, platform trial. The Lancet 2020;396:1345-52.

21. Tocilizumab, Remdesivir and Favipiravir repurposed for COVID-19: The Good, The Bad and The Ugly. . Authorea, 2020, November 30.

22. Hu H-R. Fatal Vitamin K-Dependent Coagulopathy Associated with Cefoperazone/Sulbactam: A Case Report. Drug Saf Case Rep 2019;6:6-.

23. Coronavirus: Virus isolation period extended from seven to 10 days. 2020, July 30. at https://www.bbc.com/news/uk-53588709.)

24. Li M, Chen P, Yuan Q, Song B, Ma J. Transmission characteristics of the COVID-19 outbreak in China: a study driven by data. medRxiv 2020:2020.02.26.20028431. 
25. Breakthrough: Ibuprofen/nitazoxanide/azithromycin: a battle changer personalized COVID-19 telemedicine five days protocol. 2020, June 22. at https://www.authorea.com/users/318758/articles/460853breakthrough-ibuprofen-nitazoxanide-azithromycin-a-battle-changer-personalized-covid-19-telemedicinefive-days-protocol?commit $=52337 \mathrm{e} 49 \mathrm{~b} 98 \mathrm{~b} 96 \mathrm{fae} 21$ dab166828780e5f544a80.)

26. Hong W, Chen Y, You K, et al. Celebrex Adjuvant Therapy on Coronavirus Disease 2019: An Experimental Study. Frontiers in Pharmacology 2020;11.

Figure legends

Figure 1: pre-treament CBC (patient A)

Figure 2: post-treatment CBC (patient A)

Figure 3: Pre-treatment chest CT (patient B)

Figure 4: Pre-treatment CBC (patient B)

Figure 5: Post-treatment CBC (patient B)

Figure 6: Pre-treatment chest CT (patient C)

Figure 7: Pre-treatment chest CT report (patient C)

Figure 8: pre-treament CBC (patient C)

Figure 9: post-treatment CBC (patient C) 


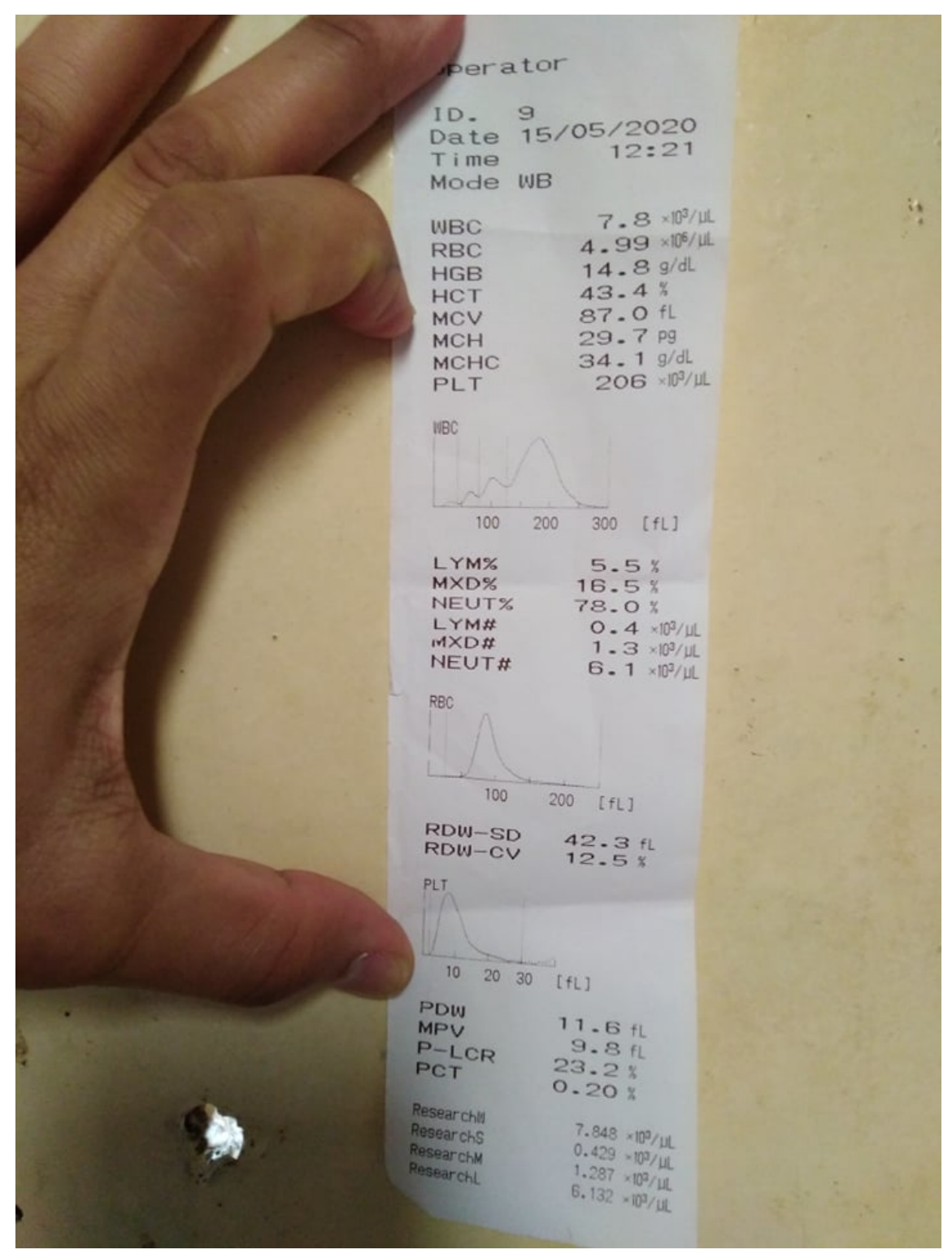

Figure 1. pre-treament CBC (patient A)

Figure 2. post-treatment CBC (patient A) 


\begin{tabular}{|c|c|c|c|c|c|}
\hline Req.Date & $23-05-2020$ & & Print.Date: & $23-05-2020$ & \\
\hline P.No & 20052303037 & Sex: & Male & Age : & 24 Years \\
\hline
\end{tabular}

\begin{tabular}{|c|c|c|c|c|c|}
\hline Teat & & Reanth & & Unit & Refirence range \\
\hline \multicolumn{6}{|l|}{ Hh\&indeses } \\
\hline Haemoglobin & & 15,6 & & $s m i d t$ & $125-17.0$ \\
\hline Haematocrit (PCV) & & 44.7 & & $\%$ & $39-49$ \\
\hline Red Cell Count & & 5.18 & & millomm & $4.3-5.7$ \\
\hline $\mathrm{MCV}$ & & 86 & & $f$ & $80-99$ \\
\hline $\mathrm{MCH}$ & & 30 & & $p g$ & 27.34 \\
\hline $\mathrm{MCHC}$ & & 35 & & $\%$ & 32.37 \\
\hline \multicolumn{6}{|l|}{ ILC \& Differential } \\
\hline & Mesult & Relatine & Ahrolite & te Result & thsolute Normal \\
\hline White cell count & 6.3 & & & Thosesund/cmm & 4.11 \\
\hline Busophils & 0 & 0.2 & 0 & /cmm & $0-100$ \\
\hline Eosinophils & 2 & 1.4 & 126 & $/ \mathrm{cmm}$ & 0.800 \\
\hline Staff & 1 & 0.6 & 63 & lamm & 0.200 \\
\hline Segmented & 48 & $37-75$ & 3024 & $\mathrm{cmm}$ & $2000-7200$ \\
\hline Lymphocytes & 43 & $20-45$ & 2709 & /cmm & $1500-3500$ \\
\hline Monocytes & 6 & $2-10$ & 378 & /cmm & $200-900$ \\
\hline \multicolumn{6}{|l|}{ PLT } \\
\hline Platelet count & & 270 & & Thausunil/cmm & $150-450$ \\
\hline MPV & & 8.6 & & $f$ & $6.5 \cdot 12$ \\
\hline PDW & & 113 & & & $9-17$ \\
\hline P.LCC & & 49 & & $x 10 \% 9 / L$ & $30-90$ \\
\hline
\end{tabular}

Comments

BLOOD PICTURE IS WITHIN NORMAL. RANGE FOR AGE \& SEX.

Figure 3: Pre-treatment chest CT (patient B) 


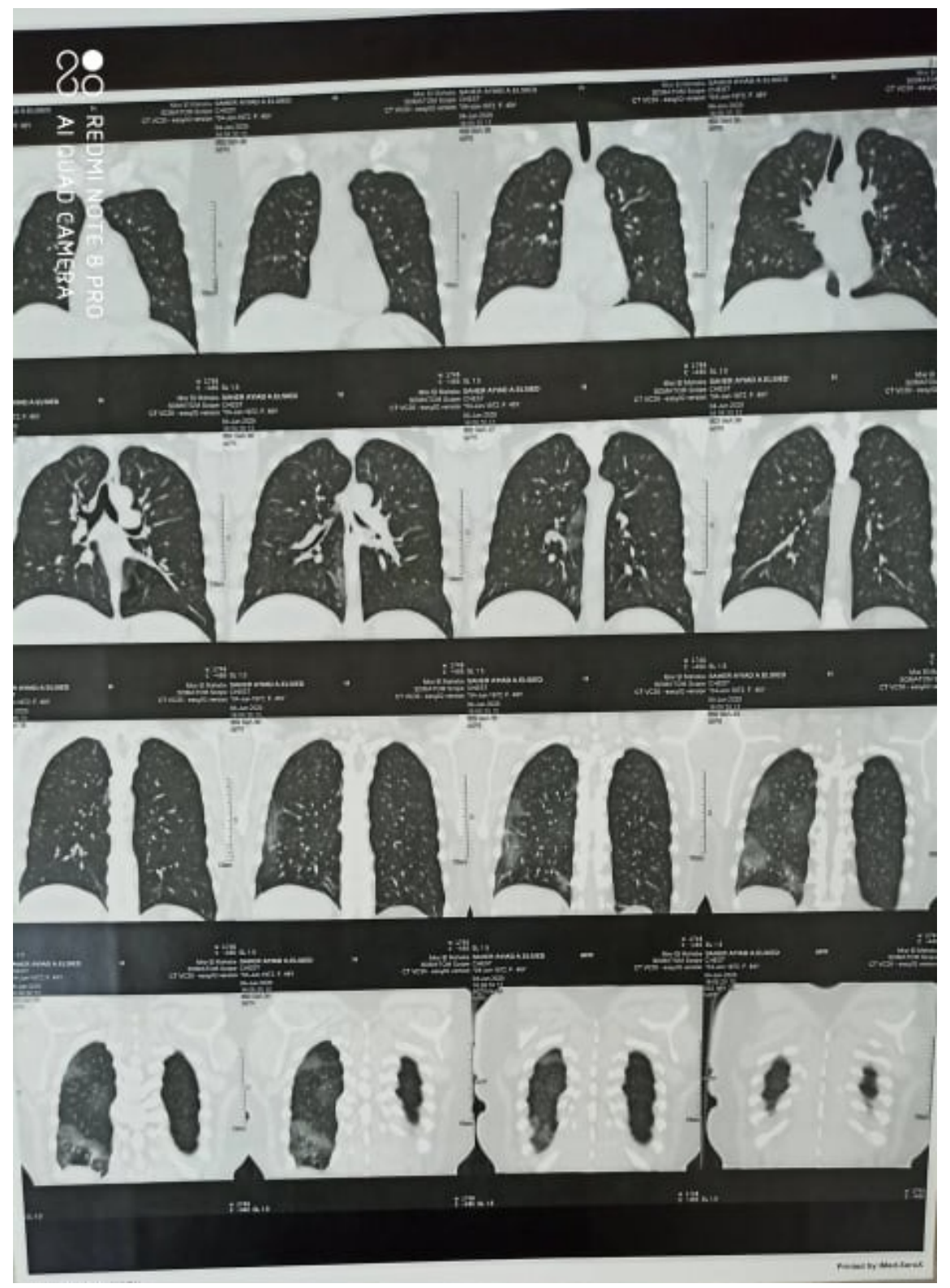

Figure 4: Pre-treatment CBC (patient B) 


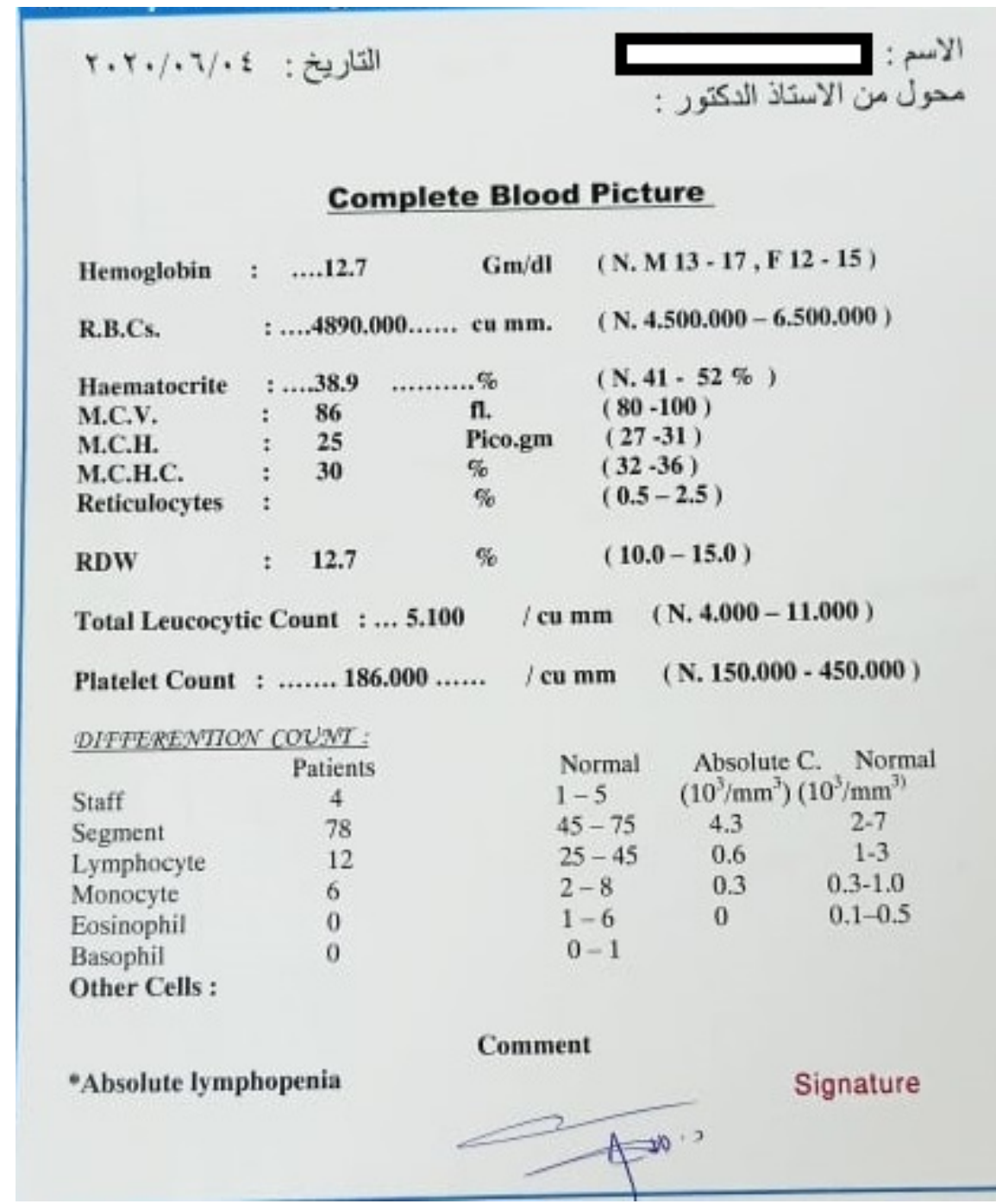

Figure 5: Post-treatment CBC (patient B) 


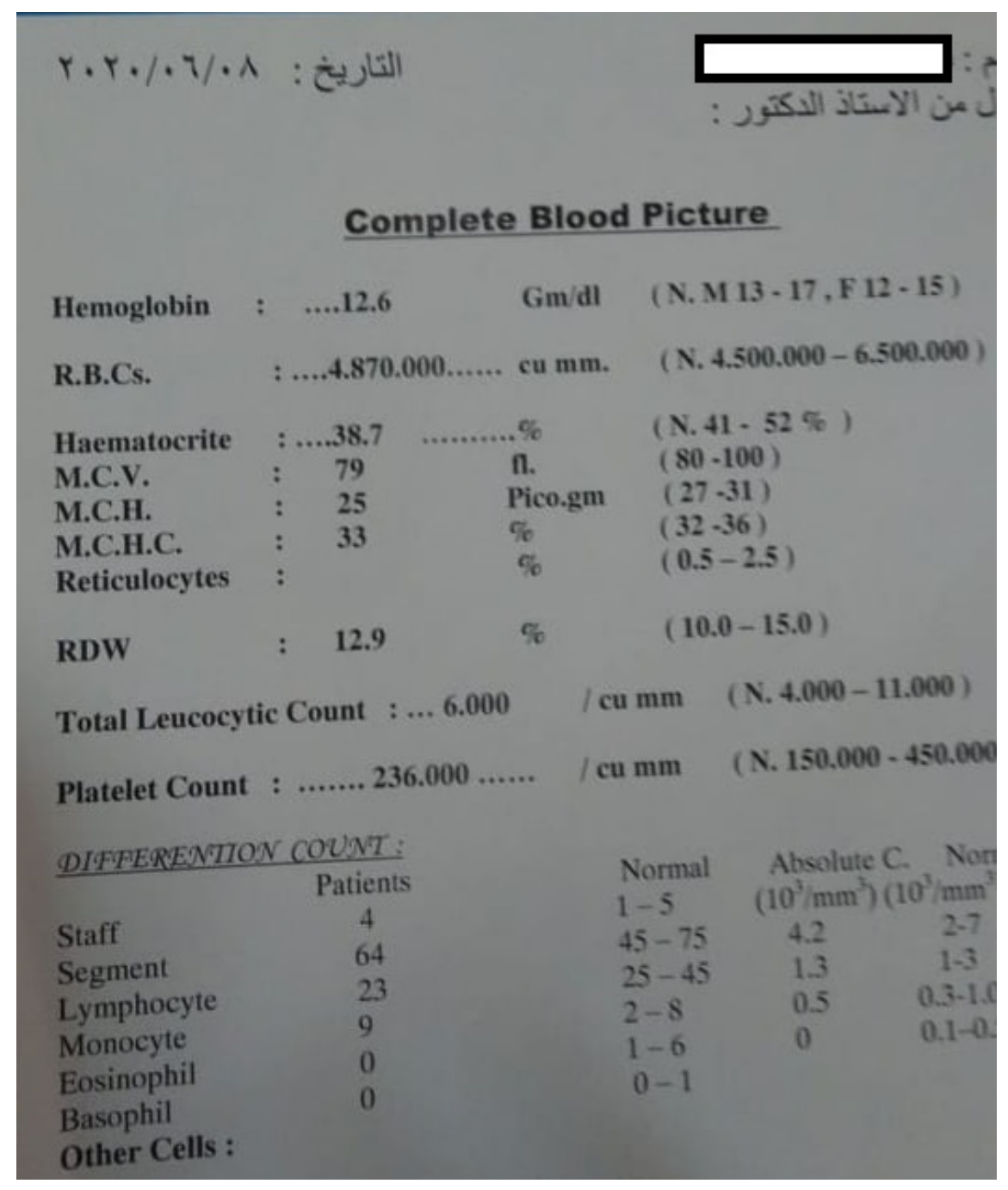

Figure 6: Pre-treatment chest CT (patient C) 


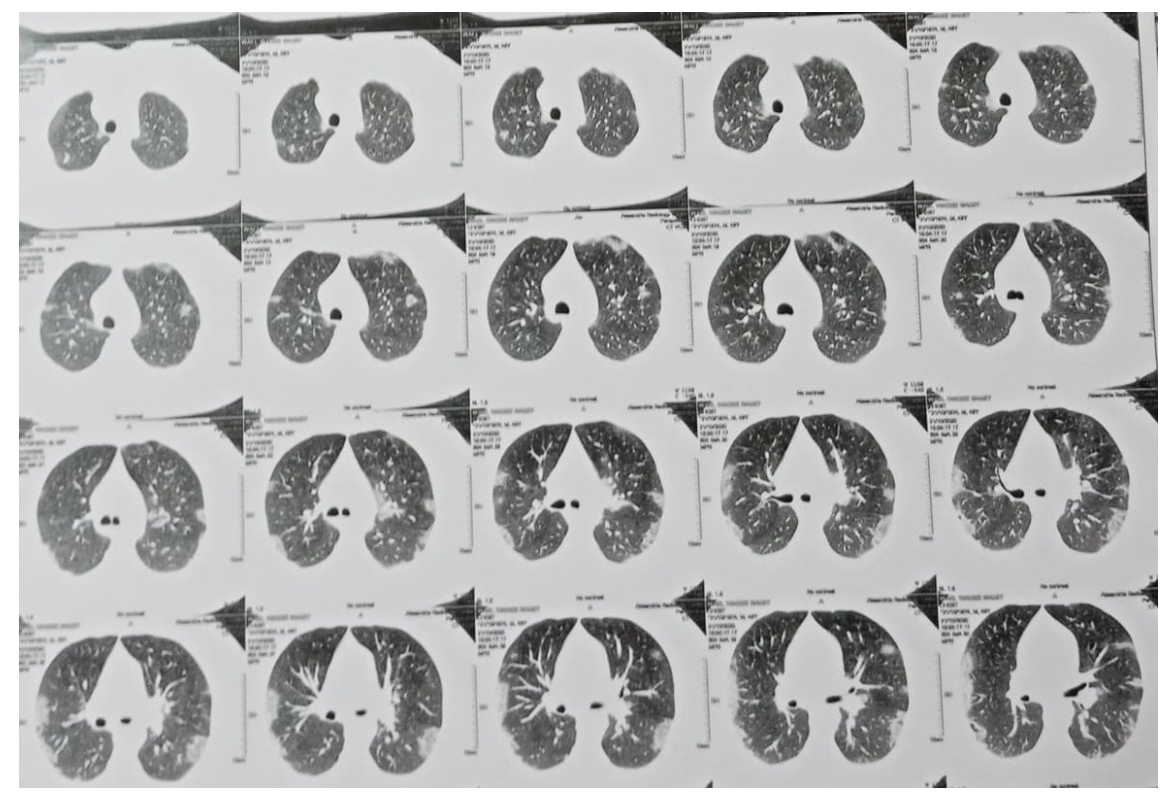

Figure 7: Post-treatment chest CT report (patient C)

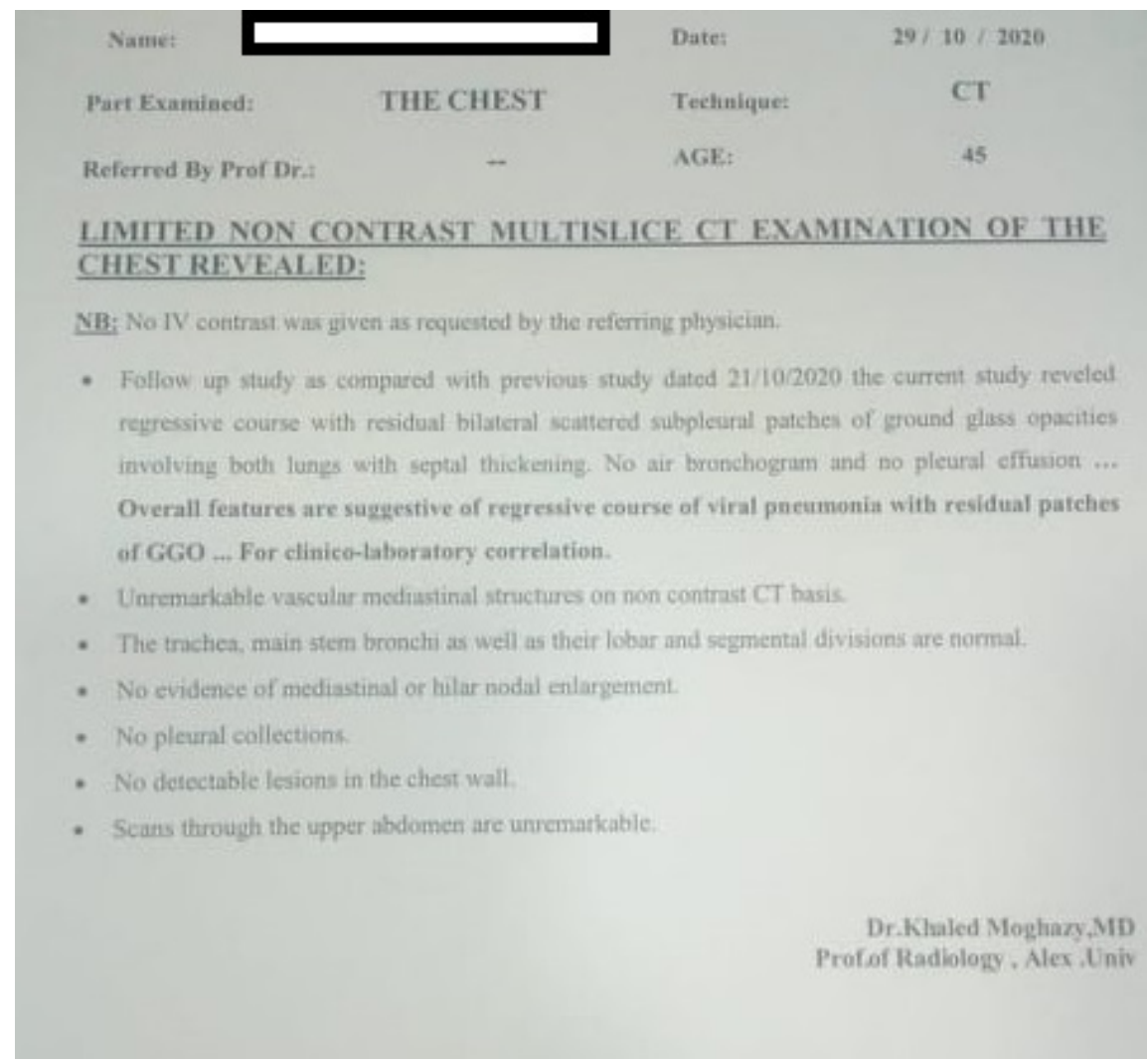

Figure 8: pre-treament CBC (patient C) 


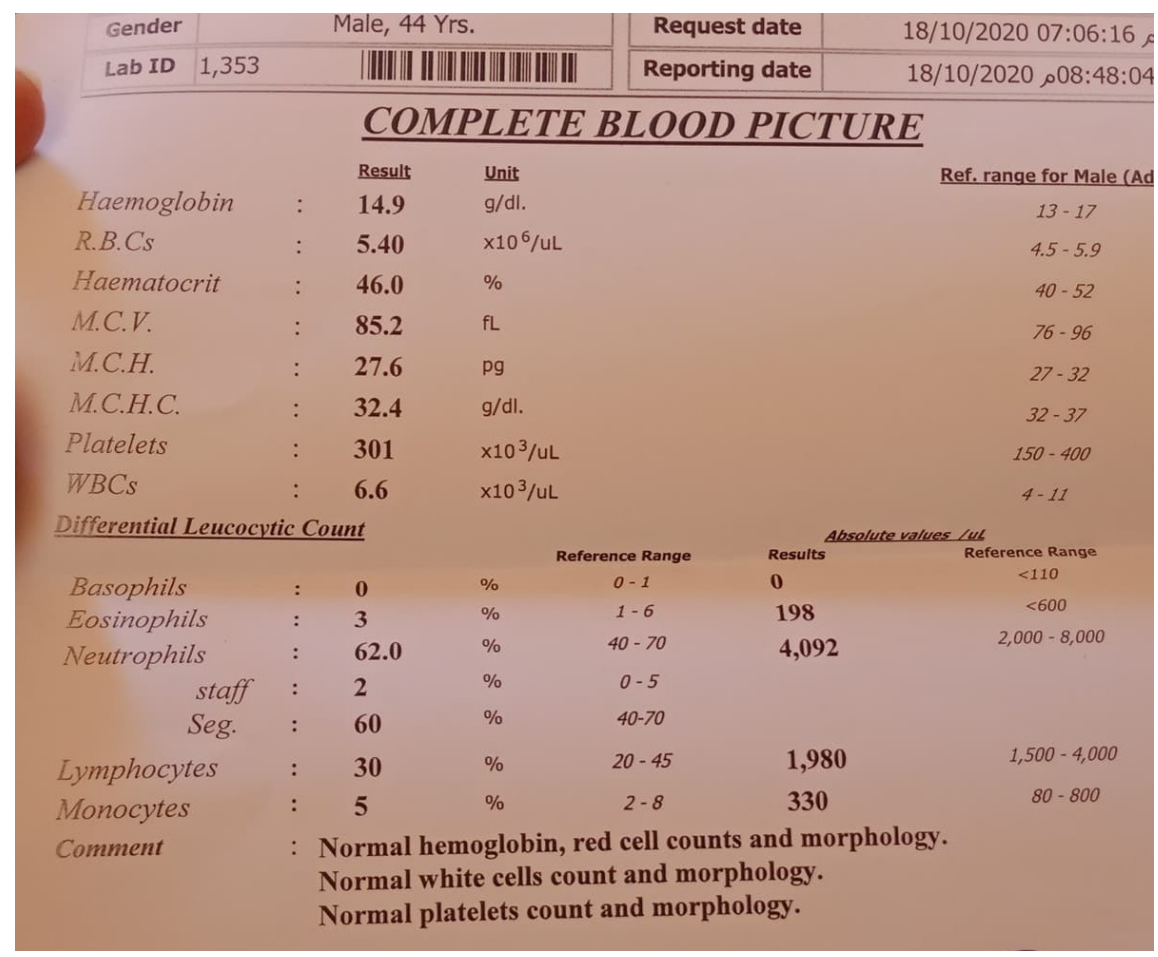

Figure 9: post-treament CBC (patient C)

\begin{tabular}{|c|c|c|c|c|c|c|c|}
\hline \multicolumn{3}{|c|}{$\begin{array}{l}\text { PATIENT NAME } \\
\text { الأستاذ| }\end{array}$} & \multicolumn{2}{|c|}{$\begin{array}{l}\text { Registered } \\
\text { Authenticated }\end{array}$} & $\begin{array}{l}25-10-2020 \text { 23:57:12 } \\
\text { 27-10-2020 08:53:34 } \\
\end{array}$ & $\begin{array}{l}\text { Collected } \\
\text { Printed }\end{array}$ & $\begin{array}{l}26-10-2020 \text { 15:27:02 } \\
27-10-2020 \text { 10:06:19 } \\
\end{array}$ \\
\hline Visit Number & Age & Gender & \multicolumn{3}{|c|}{ Referred By } & \multicolumn{2}{|r|}{ Client ID } \\
\hline 33920515955 & 45 Year & Male & \multicolumn{3}{|r|}{ Prof : - } & & 30129 \\
\hline \multicolumn{3}{|l|}{ Test Name } & \multicolumn{2}{|l|}{ Result } & Unit & \multicolumn{2}{|c|}{ Reference Range } \\
\hline \multicolumn{8}{|c|}{ Complete Blood Picture } \\
\hline \multicolumn{3}{|l|}{ Haemoglobin } & \multicolumn{2}{|l|}{14.7} & $g / d l$ & \multicolumn{2}{|l|}{$12.5-17.5$} \\
\hline \multicolumn{3}{|l|}{ Haematocrit (PCV) } & \multicolumn{2}{|l|}{45.5} & $\%$ & \multicolumn{2}{|l|}{$41-52$} \\
\hline \multicolumn{3}{|l|}{ RBCs Count } & \multicolumn{2}{|l|}{5.18} & Millions / cmm & \multicolumn{2}{|l|}{$4.5-5.9$} \\
\hline \multicolumn{3}{|l|}{ MCV } & \multicolumn{2}{|l|}{87.8} & fl & \multicolumn{2}{|l|}{$80-100$} \\
\hline \multicolumn{3}{|l|}{$\mathrm{MCH}$} & \multicolumn{2}{|l|}{28.4} & pg & $27-33$ & \\
\hline MCHC & & & 32.3 & & $g / d l$ & $31-37$ & \\
\hline RDW-CV & & & 12.2 & & $\%$ & $11.5-15$ & \\
\hline Platelet Count (EDT & od) & & 407 & & thousands $/ \mathrm{cmm}$ & $150-450$ & \\
\hline Total Leucocytic Col & DTA Blood & & 7.3 & & thousands / cmm & $4-11$ & \\
\hline & Percen & Values & & Absolu & te Values & & \\
\hline Neutrophils & 38.2 & $\%$ & & 2.78 & x10^9/L & $2-7$ & \\
\hline Staff & 4 & $\%$ & & 0.29 & $\times 10^{\wedge} 9$ & & \\
\hline Segmented & 34.2 & $\%$ & & 2.50 & $x 10^{\wedge} 9$ & & \\
\hline Lymphocytes & 47.9 & $\%$ & & 3.50 & x10^9/L & $1-4.8$ & \\
\hline Monocytes & 10.7 & $\%$ & & 0.78 & $\times 10^{\wedge} 9 / L$ & $0.2-1$ & \\
\hline Eosinophils & 2.7 & $\%$ & & 0.20 & x10^9/L & $0.1-0.45$ & \\
\hline Basophils & 0.5 & $\%$ & & 0.04 & x10^9/L & $0-0.1$ & \\
\hline Other Cells & & & & & & & \\
\hline Comment: & & & & & & & \\
\hline $\begin{array}{l}\text { RELATIVE LYMPHOC } \\
\text { RELATIVE MONOCYT } \\
\text { Follow up is recomm }\end{array}$ & & & & & & & \\
\hline
\end{tabular}

